\title{
Magnetization curves and hysteresis loops of nanostructured rare earth titanates
}

\author{
Anatoly B. Rinkevich ${ }^{1, *}$, Alexander V. Korolev ${ }^{1}$, Mikhail I. Samoilovich ${ }^{2}$, Sergej O. Demokritov ${ }^{1,3}$, and Dmitry V. Perov ${ }^{1}$ \\ ${ }^{1}$ M.N. Miheev Institute of Metal Physics, 620108 S. Kovalevskaya st. 18, Ekaterinburg, Russia \\ ${ }^{2}$ Central Research Technological Institute "TECHNOMASH", 121108 I. Franko st. 4, Moscow, Russia \\ ${ }^{3}$ Institute for Applied Physics and Center for Nonlinear Science, University of Muenster, 48149 Corrensstrasse 2-4, Muenster, Germany
}

\begin{abstract}
Magnetic properties of the nanocomposite materials containing particles of rare earth titanates with pyrochlore structure have been investigated. For the nanocomposites with $\mathrm{Gd}_{2} \mathrm{Ti}_{2} \mathrm{O}_{7}, \mathrm{La}_{2} \mathrm{Ti}_{2} \mathrm{O}_{7}$ it has been observed that the effective magnetic moment in the nanocomposites differs substantially from that for $\mathrm{Gd}^{3+}$ and $\mathrm{La}^{3+}$ ions. The hysteresis loops was obtained for the nanocomposites with $\mathrm{Dy}^{3+}, \mathrm{Gd}^{3+}, \mathrm{Yb}^{3+}, \mathrm{Er}^{+}$, $\mathrm{Sm}^{3+}$ ions. There is no hysteresis loops for the nanocomposites with $\mathrm{La}_{2} \mathrm{Ti}_{2} \mathrm{O}_{7}, \mathrm{Pr}_{2} \mathrm{Ti}_{2} \mathrm{O}_{7}$ and $\mathrm{Nd}_{2} \mathrm{Ti}_{2} \mathrm{O}_{7}$ particles. It has been shown that nanocomposites with $\mathrm{Yb}_{2} \mathrm{Ti}_{2} \mathrm{O}_{7}, \mathrm{Dy}_{2} \mathrm{Ti}_{2} \mathrm{O}_{7}$ and $\mathrm{Er}_{2} \mathrm{Ti}_{2} \mathrm{O}_{7}$ particles have one crossing point on the descending branches of hysteresis loop in some temperature range.
\end{abstract}

\section{Introduction}

Investigation of low temperature magnetic properties of rare earth titanantes with the pyrochlore structure constitutes a fashionable task. Pyrochlore titanates have the type of $\mathrm{R}^{3+}{ }_{2} \mathrm{Ti}^{4+}{ }_{2} \mathrm{O}_{7}$ where $\mathrm{R}^{3+}$ is a rare-earth ion. They potentially promote to constitute a geometrically frustrated magnet. Short range ordered structures such as spin glasses, spin ices and spin liquids are organized in these systems instead of long range ordered ground states [1]. The problem of "spin ice" established for several titanates attracts the most interest [2].

The aim of this paper is to investigate the magnetization curves and hysteresis loops for several nanocomposite rare earth titanates of $\mathrm{R}_{2} \mathrm{Ti}_{2} \mathrm{O}_{7}$ type. The size of the particles inside the nanocomposites varied from 5 to $60 \mathrm{~nm}$. The particles are composed of La, Dy, $\mathrm{Yb}, \mathrm{Er}, \mathrm{Sm}, \mathrm{Nd}, \mathrm{Gd}$ and $\mathrm{Pr}$ titanates. Major and minor magnetic-hysteresis loops have been measured in temperature range from 2 to $50 \mathrm{~K}$. We observed that for several nanocomposites the descending branches of the hysteresis loops measured at different temperatures cross at one point of the magnetization $M$ - magnetic field $H$ plane.

\section{Preparation of samples and their structure}

Sintering of monodisperse opal matrices with the submicron $\mathrm{SiO}_{2}$ spheres is described elsewhere [3]. The nanocomposite materials with embedded titanate nanoparticles are obtained by chemical synthesis method with posterior thermal treatment. Annealing was carried out at temperature $900{ }^{\circ} \mathrm{C}$, whereas impregnations and afterheats were repeated 5 times resulting in gradual infill of the inter-sphere voids. The SEM image of the 3D-nanocomposites structure is shown in Fig.1. The inserted particles have polycrystalline structure and irregular shape. The most part of the particles has the size from 5 to $60 \mathrm{~nm}$. The important feature seen in Fig.1, where structure of the nanocomposite with $\mathrm{Yb}_{2} \mathrm{Ti}_{2} \mathrm{O}_{7}+\mathrm{TiO}_{2}$ particles is shown, that the particles form aggregates, where individual particles are located very close one to another, at the distances of few nanometers.

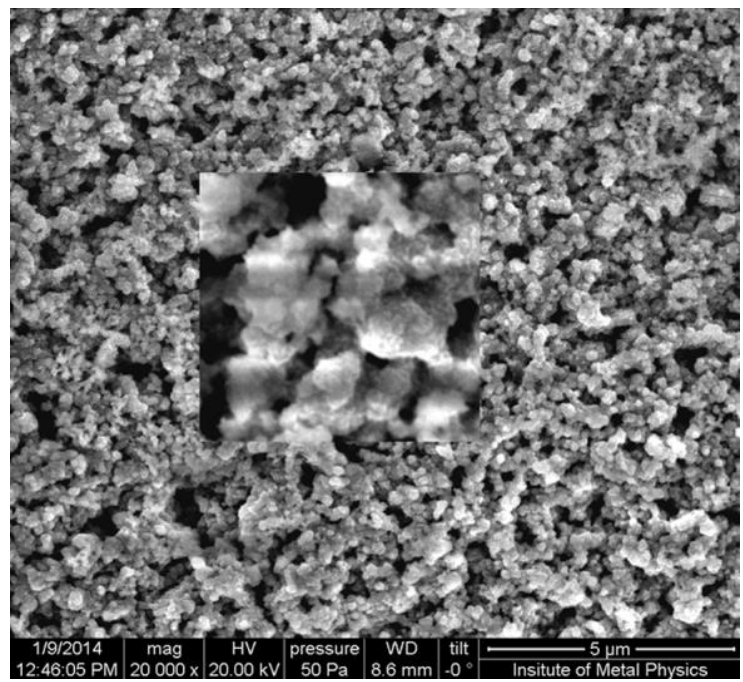

Fig. 1. Sampling electron microscope image of the nanocomposite contained particles of $\mathrm{Yb}_{2} \mathrm{Ti}_{2} \mathrm{O}_{7}+\mathrm{TiO}_{2}$, magnification $\mathrm{x} 20000$. 
The following scheme of the sample enumeration is accepted. In the sample number No.244/5-900, for example, at first the sequential number of the sample (244) is indicated and then the impregnations number goes (5), and then the temperature of heat treatment $(900$ ${ }^{\circ} \mathrm{C}$ ) is shown. Concluding this section we note that the samples with nanocomposite rare earth titanates have desirable chemical composition and phase content.

\section{Magnetization curves and magnetic moments}

Magnetic properties of the nanocomposites were studied using the PPMS-9 system. The magnetization curves and hysteresis loops were measured in magnetic fields to 30 $\mathrm{kOe}$ at temperatures from 2 to $20 \mathrm{~K}$. The magnetization curves for sample No. 244/5-900 with particles of $\mathrm{Gd}_{2} \mathrm{Ti}_{2} \mathrm{O}_{7}$, which have been measured at different temperatures, are shown in Fig.2a. This figure presents also the appropriate theoretical dependencies calculated from Brillouin formula [4]

$$
M=N g \mu_{B} J B_{J}(x),
$$

where

$$
B_{J}(x)=\frac{2 J+1}{2 J} \operatorname{cth}\left(\frac{2 J+1}{2 J} x\right)-\frac{1}{2 J} \operatorname{cth} \frac{x}{2 J},
$$

$M$ is the magnetic moment, $N$ is the number of magnetic atoms in the sample, $\mu_{B}$ is the Bohr magneton, $J$ is the total angular momentum quantum number, $x=\frac{g \mu_{B} J H}{k_{B} T}$, $g$ is the Landé factor, $H$ is external magnetic field, $k_{B}$ is the Boltzmann constant, $T$ is temperature.

The effective magnetic moment per one rare earth atom in a sample is calculated by the following way

$$
\mu=g \mu_{B} \sqrt{J(J+1)} .
$$

The approximate curves shown in Fig.2a are corresponding to the quantum number $J=7 / 2$ and effective magnetic moment $\mu=7.94 \mu_{B}$.

Approximation of the experimental dependences is realized via variation of $J$ at a constant value of $g$. It is clearly seen from Fig.2a that the magnetization curves measured at different temperatures, differ markedly from each other. These data evidently proves interaction either between $\mathrm{Gd}^{3+}$ ions or between the particles. Because of this, the approximation of the experimental magnetization curves measured at different temperatures has been performed, in order to get the temperature variation of effective magnetic moment. The results are summarized in Fig. 2b. It is easy to verify that for $\mathrm{Gd}_{2} \mathrm{Ti}_{2} \mathrm{O}_{7}$ the obtained from approximation values $\mu$ varying from 3.92 to 4.81 , and also $J$ varying from 1.96 to 2.4 are much close to ${ }^{5} \mathrm{~S}_{2}$ term, than to ${ }^{8} \mathrm{~S}_{7 / 2}$ term which is generally recognized for $\mathrm{Gd}^{3+}$ ion. The effective magnetic moment deduced from this approximation for the nanocomposite with $\mathrm{Gd}_{2} \mathrm{Ti}_{2} \mathrm{O}_{7}$ particles $\mu=4.81 \mu_{B}$ at $\mathrm{T}=10 \mathrm{~K}$ differs substantially from the effective magnetic moment of ion $\mu=7.94 \mu_{B}$ [4]. Moreover, it is worthy to mention that the effective magnetic moment in nanocomposite increases with temperature, see Fig.2b.
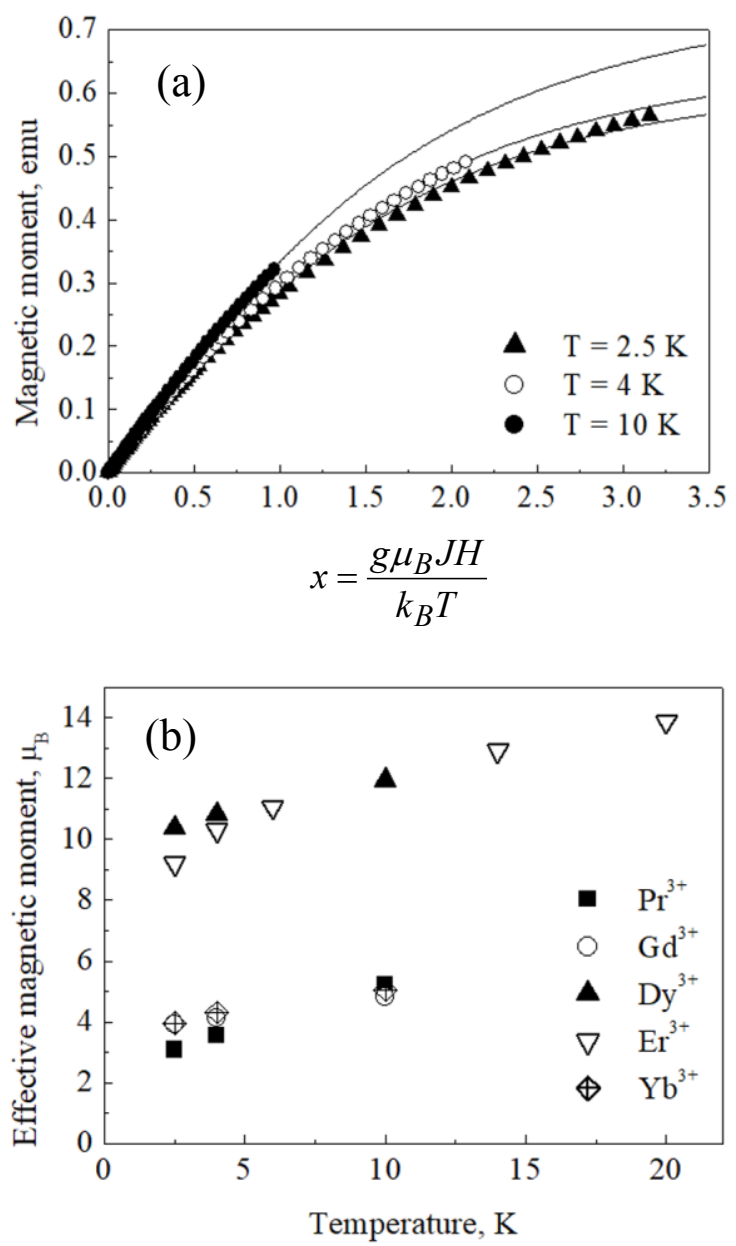

Fig. 2. Magnetization curves for the sample No.244/5900 with particles of $\mathrm{Gd}_{2} \mathrm{Ti}_{2} \mathrm{O}_{7}$ measured at different temperatures - (a); effective magnetic moment per rare earth ion for several nanocomposite pyrochlore titanates with different rare earth ions - (b).

The hysteresis loops were obtained for the nanocomposites with $\mathrm{Yb}_{2} \mathrm{Ti}_{2} \mathrm{O}_{7}, \mathrm{Dy}_{2} \mathrm{Ti}_{2} \mathrm{O}_{7}, \mathrm{Er}_{2} \mathrm{Ti}_{2} \mathrm{O}_{7}$ and $\mathrm{Sm}_{2} \mathrm{Ti}_{2} \mathrm{O}_{7}$ particles at cryogenic temperatures. The narrow hysteresis loops for the sample No.228/5-900 with particles of $\mathrm{Yb}_{2} \mathrm{Ti}_{2} \mathrm{O}_{7}+\mathrm{TiO}_{2}$ measured at different temperatures are shown in Fig.3. The hysteresis loops for the sample No.230/5-900 with particles of $\mathrm{Er}_{2} \mathrm{Ti}_{2} \mathrm{O}_{7}$ measured at two temperatures $2 \mathrm{~K}$ and $10 \mathrm{~K}$ are shown in Fig.4a. The presence of hysteresis loop itself could be caused by two reasons: 1 . Influence of admixture of $3 \mathrm{~d}$ elements leading to magnetic polarization and magnetic ordering of the nanocomposite. 2. Intrinsic magnetic polarization comes true because of nano-scale size of titanate particles. The hysteresis loop is observed for the samples of several different titanates in which the 
admixture of $3 \mathrm{~d}$ elements does not found. Because of this circumstance and a fact that the presence of defects in the nanocomposites can stabilize the magnetic structure, the second reason seems to be most probable one. Moreover, confinement for the movement of monopoles, which are quasi-particles in spin ice solids [5], inside a nanoparticle can be a valuable factor for nanocomposite samples to get magnetic ordering. The descending branches of hysteresis loops have been studied in detail, see Fig.4b for example. For several nanocomposites it has been obtained experimentally that discussed descending branches measured at different temperatures intersect each other in one point at $M-H$ plane. This fact has been established for nanocomposite titanates with $\mathrm{Yb}_{2} \mathrm{Ti}_{2} \mathrm{O}_{7}, \mathrm{Dy}_{2} \mathrm{Ti}_{2} \mathrm{O}_{7}, \mathrm{Er}_{2} \mathrm{Ti}_{2} \mathrm{O}_{7}$ particles. For nanocomposite with $\mathrm{Sm}_{2} \mathrm{Ti}_{2} \mathrm{O}_{7}$ particles the area has been defined where these dependences intersect. The hysteresis loop is lacking for the nanocomposites with $\mathrm{La}_{2} \mathrm{Ti}_{2} \mathrm{O}_{7}, \mathrm{Pr}_{2} \mathrm{Ti}_{2} \mathrm{O}_{7}$ and $\mathrm{Nd}_{2} \mathrm{Ti}_{2} \mathrm{O}_{7}$ particles.

In the presence of the point or region of intersection of the descending parts of hysteresis loops there should be a temperature interval within which the magnetization measured at the intersection point remains constant or only slow temperature dependent. The temperature dependences of magnetization for sample No.243a/5-900 with $\mathrm{Sm}_{2} \mathrm{Ti}_{2} \mathrm{O}_{7}$ particles measured in magnetic field of $H$ $=-100 \mathrm{Oe}$, that is the field of the intersection region, and the remanent magnetization $M_{r}$, are shown in Fig.5. It is clearly seen that these dependences are quite different at low temperatures, Namely, the dependence measured in the field of $H=-100$ Oe has an interval from $2 \mathrm{~K}$ to $5 \mathrm{~K}$ where magnetization varies slowly, and this interval is lacking for $M_{r}$. The temperature dependence of coercive field for samples with the point or region of intersection are expected to be unusual. This dependence for sample No.243a/5-900 with $\mathrm{Sm}_{2} \mathrm{Ti}_{2} \mathrm{O}_{7}$ particles is presented in Fig.5b. This dependence contains a low temperature section where the coercive field decreases if temperature diminishes.

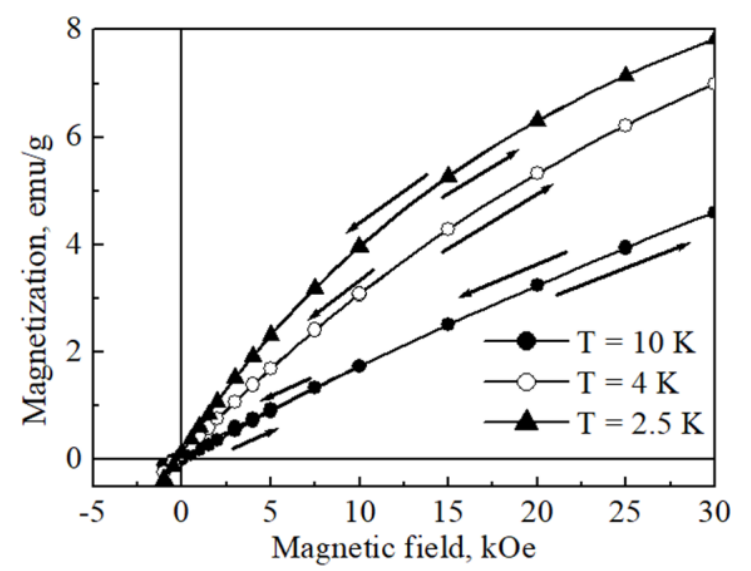

Fig. 3. Hysteresis loops for the sample No.228/5-900 with particles of $\mathrm{Yb}_{2} \mathrm{Ti}_{2} \mathrm{O}_{7}+\mathrm{TiO}_{2}$ measured at different temperatures.
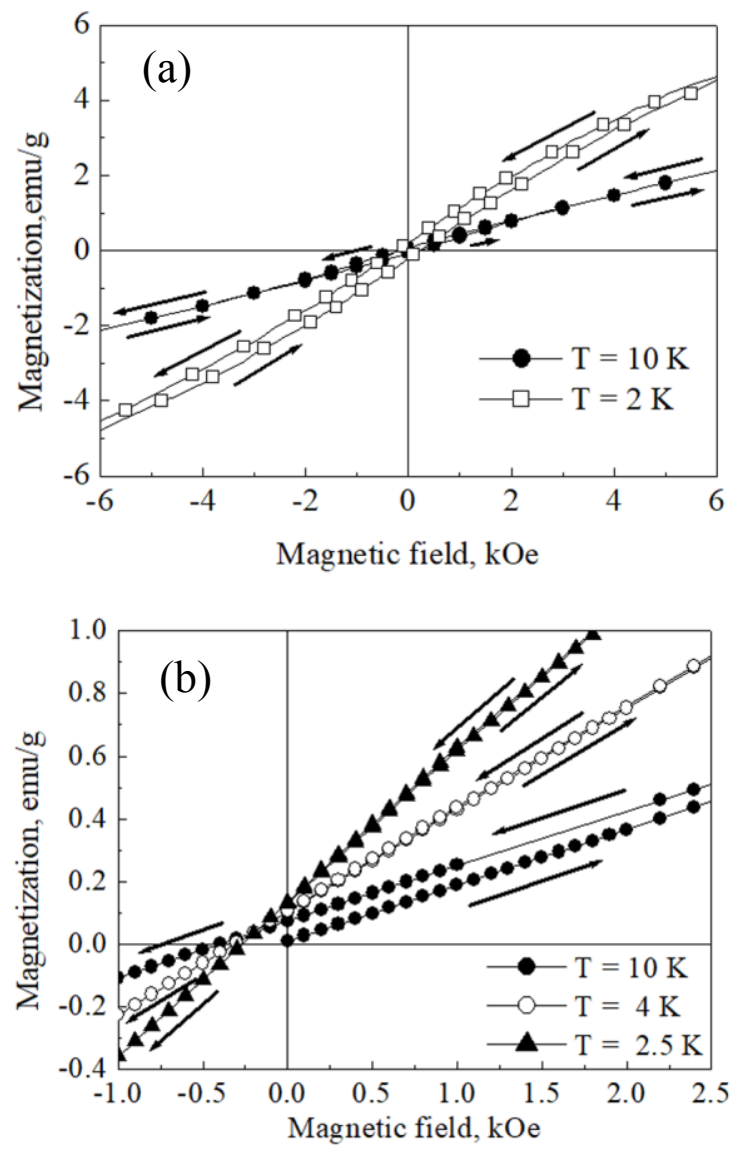

Fig. 4. Hysteresis loops for the sample No.230/5-900 with particles of $\mathrm{Er}_{2} \mathrm{Ti}_{2} \mathrm{O}_{7}$ measured at two temperatures - (a); part of the hysteresis loop for the sample No.228/5-900 with particles of $\mathrm{Yb}_{2} \mathrm{Ti}_{2} \mathrm{O}_{7}+\mathrm{TiO}_{2}$ showing the crossing point - (b).

\section{Conclusions}

Magnetic properties of nanocomposite titanates of $\mathrm{R}_{2} \mathrm{Ti}_{2} \mathrm{O}_{7}$ type are investigated where $\mathrm{R}$ is a rare earth ion: $\mathrm{Nd}^{3+}, \mathrm{La}^{3+}, \mathrm{Pr}^{3+}, \mathrm{Sm}^{3+}, \mathrm{Gd}^{3+}, \mathrm{Dy}^{3+}, \mathrm{Er}^{3+}, \mathrm{Yb}^{3+}$. Magnetization curves of titanates are carefully studied and it is found that the magnetization curve is well approximated by Brillouin function. The effective magnetic moment of most titanates under study is to a certain extent different from the moment of subsequent ion. For nanocomposite titanate $\mathrm{Gd}_{2} \mathrm{Ti}_{2} \mathrm{O}_{7}$, however, obtained quantum number $J$ and effective magnetic moment $\mu$ are markedly different from the values for the ion. The effective magnetic moment of all studied titanates increases when temperature increases. A high density of crystallographic defects inevitably presents in the nanoparticles, in particular at their subsurface layers. Consequently, due to this reason a finite-size induced magnetic ordering should be expected. 

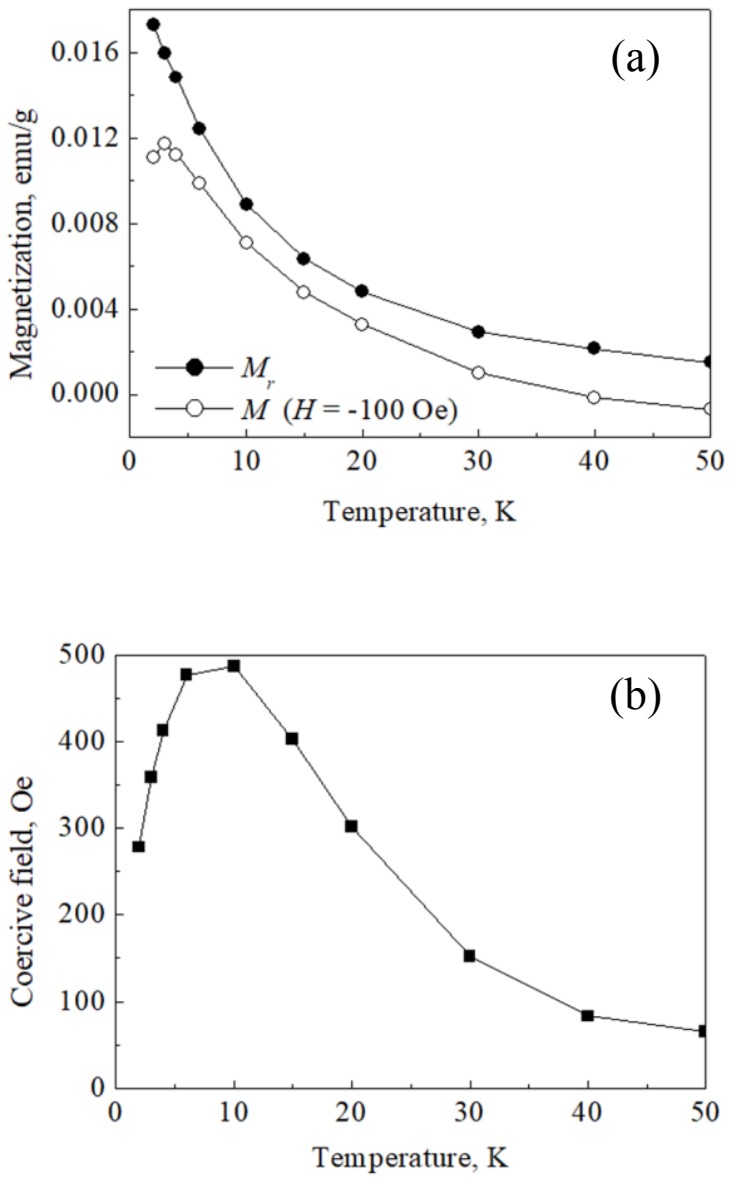

Fig. 5. Temperature dependences of the magnetization for sample No.243a/5-900 with $\mathrm{Sm}_{2} \mathrm{Ti}_{2} \mathrm{O}_{7}$ measured in magnetic field of $H=-100 \mathrm{Oe}$, that is close to the field of the intersection region, and the remanent magnetization $M_{r}-$ (a); temperature dependence of coercive field for the same sample - (b).

The hysteresis loops were obtained for the nanocomposites with $\mathrm{Yb}_{2} \mathrm{Ti}_{2} \mathrm{O}_{7}, \mathrm{Dy}_{2} \mathrm{Ti}_{2} \mathrm{O}_{7}, \mathrm{Er}_{2} \mathrm{Ti}_{2} \mathrm{O}_{7}$ and $\mathrm{Sm}_{2} \mathrm{Ti}_{2} \mathrm{O}_{7}$ particles at cryogenic temperatures. The hysteresis loop is lacking for the nanocomposites with $\mathrm{La}_{2} \mathrm{Ti}_{2} \mathrm{O}_{7}, \mathrm{Pr}_{2} \mathrm{Ti}_{2} \mathrm{O}_{7}$ and $\mathrm{Nd}_{2} \mathrm{Ti}_{2} \mathrm{O}_{7}$ particles. For several nanocomposites it has been obtained experimentally that discussed descending branches measured at different temperatures intersect each other in one point at $M-H$ plane. In the presence of the point or region of intersection of the descending parts of hysteresis loops there is a temperature interval within which the magnetization measured at the intersection point remains constant or only slow temperature dependent.
The work is carried out within the RAS theme "Spin" No. 01.2.006 01201463330, with partial support of grant from Russian Ministry of Education and Science No. 14.Z50.31.0025 and RFBR grant No. 17-02-00029. Electron microscopic studies and magnetic measurements are carried out in the Testing Center of Institute of Metal Physics.

\section{References}

1. J.S. Gardner, M.J.P. Gingras, J.E. Greedan, Reviews of Modern Physics 82, 53-107 (2010)

2. S.T. Bramwell, M.J.P. Gingras, Science 294, 1495-1501 (2001)

3. A.B. Rinkevich, A.M. Burkhanov, M.I. Samoilovich, A.F. Belyanin, S.M. Kleshcheva, E.A. Kuznetsov, Russ. J. Gen. Chem. 83, 21482158 (2013)

4. J.S. Smart, Effective Field Theories of Magnetism (W.B. Saunders Company, Philadelphia-London, 1966)

5. C. Castelnovo, R. Moessner, S.L. Sondhi, Nat. Lett. 451, 42-45 (2008) 